\section{Radiologische Diagnostik beim Nager-Syndrom}

Das Nager-Syndrom zeichnet sich durch die Kombination von kraniofazialer Dysostose und begleitenden Extremitätenanomalien aus. Von diesem erstmals von Nager und de Reynier 1948 beschriebenen Syndrom sind aus der Literatur bislang ca. 70 Fälle bekannt. $\mathrm{Zu}$ den klinischen Leitsymptomen zählen Unterlidkolbome, ein antimongoloider Lidachsenverlauf, eine Ohrmuscheldysplasie mit Gehörgangsatresie, eine Aplasie des Kiefergelenkes sowie eine Hypoplasie des Ramus ascendens mandibulae. Als Extremitätenanomalien treten zusätzlich eine Daumenhypoplasie oder -aplasie, eine Hypoplasie von Radius und Ulna sowie eine Verkürzung des Humerus auf. Ursächlich liegt eine Genmutation mit unregelmäßig autosomal dominantem Erbgang vor.

\section{Fallbeschreibung}

Fall 1

Ein neugeborener Junge mit Nager-Syndrom wurde der radiologischen Abteilung zur präoperativen Planung bei ausgeprägter Unterkieferhypoplasie zugewiesen. Bereits in der 33. SSW fielen bei einer Ultraschalluntersuchung eine Verkürzung der Unterarme und ein Pes equinovarus rechts auf. Postpartum zeigte sich neben der Unterkieferhypoplasie eine zusätzliche Aplasie des weichen Gaumens. Als Folge kam es unmittelbar postpartum zu rezidivierenden Atempausen, so dass die Anlage eines tiefen pharyngealen Tubus mit CPAP-Beatmung notwendig wurde. $\mathrm{Zu}-$ sätzlich traten erhebliche Schluckstörungen auf. Die konventionellen Röntgenaufnahmen der Extremitäten bestätigten einen mesomalen Minderwuchs. An der rechten Hand fehlte der 2. Strahl und der linke Daumen war nur rudimentär angelegt.

Zur Klärung der „Felsenbeinsituation“ und zur Planung einer Distraktionsosteogenese des Unterkiefers wurde eine Mehrschicht-Spiral-CT (MSCT) durch-

Fortschr Röntgenstr 2001; 173: 1147-1148 (C) Georg Thieme Verlag Stuttgart · New York ISSN 1438-9029 geführt. Dabei wurden eine Schichtdicke von 1,25 mm, ein Pitch von 3 bei einer Tischvorschubgeschwindigkeit von $3,75 \mathrm{~mm}$ und ein Röhrenstrom von 50 mAs gewählt. Zur Anfertigung multiplanarer Reformationen und eines Oberflächenmodels erwies sich ein Rekonstruktionsintervall von $0,6 \mathrm{~mm}$ als günstig.

Die CT-Untersuchung des Gesichtsschädels bestätigte eine ausgeprägte Retround Mikrognathie. Der Ramus ascendens mandibulae war hypoplastisch, aber symmetrisch angelegt und zeigte einen Kieferwinkel von etwa $170^{\circ}$ gegenüber der Norm von $110-140^{\circ}$ (Abb.1). Darüber hinaus lag eine dysplastische $\mathrm{Na}$ senhaupthöhle mit Choanalenge rechts vor. Der äußere Gehörgang war beidseits knöchern verschlossen und das Mittelohr jeweils nur partiell angelegt (Abb. 2).

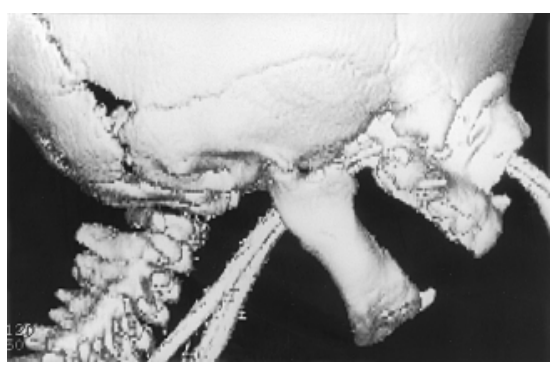

Abb. 1 Seitliche Darstellung eines 3D-Oberflächenmodells auf Basis eines MSCT des Mittelgesichtes bei Nager-Syndrom. Abgeflachter Kiefergelenkwinkel und Mikrognathie.

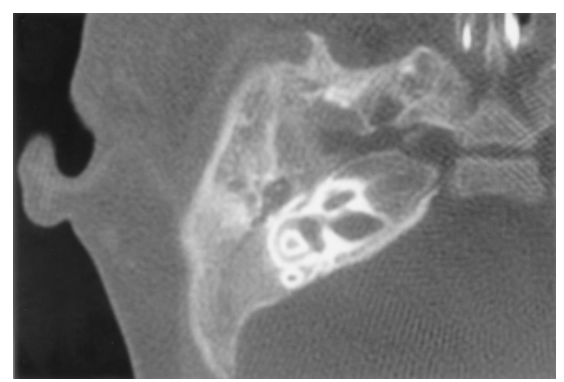

Abb. 2 MSCT des rechten Felsenbeins bei Nager-Syndrom mit Nachweis einer knöchernen Gehörgangsatresie.
Fall 2

Hierbei handelte es sich um ein Mädchen einer Erstgravida, das nach unauffälligem Schwangerschaftsverlauf spontan geboren wurde. Auch hier kam es zu postpartalen Atemstörungen bei insuffizienter Spontanatmung aufgrund einer Dysostosis mandibularis. Zusätzlich lagen eine knöcherne Gehörgangsatresie, eine Spalte des weichen Gaumens, eine radioulnare Synostose mit Verkürzung beider Unterarme und eine Daumenaplasie rechts sowie Daumenhypoplasie links vor. Zur präoperativen Planung erfolgte ebenfalls eine MSCT mit Anfertigung von entsprechenden Oberflächenrekonstruktionen. Auf dieser Basis wurde eine Distraktionsosteogenese mit Unterkieferosteotomie und Extension durchgeführt (Abb.3), wobei nach Abschluss der Behandlung eine Wiederherstellung der Unterkieferkonfiguration mit Spontanatmung erreicht wurde.

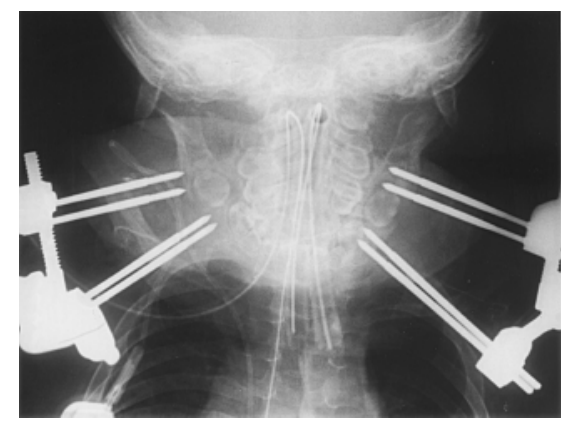

Abb. 3 Zustand bei Distraktionsosteogenese bei einem 1-jährigen Patienten mit Nager-Syndrom.

\section{Diskussion}

Das Nager-Syndrom wird als Folge eines heterogenen Felddefektes betrachtet, der auf Störungen in der 3. und 4. Embryonalwoche zurückzuführen ist (Witkowski et al., Lexikon der Syndrome und Fehlbildungen, Springer, S. $701-$ 703). Eine endgültige Klärung der Ätiologie dieser angeborenen Fehlbildung steht allerdings noch aus. Zumeist tritt das Syndrom sporadisch auf (Stephan MJ et al., Am J med Genet 1990; 35: 493). Das Wiederholungsrisiko für Verwandte eines Merkmalträgers kann im Hinblick auf die unklare Ätiologie vorläufig nur empirisch eingeschätzt werden und ist für Geschwister lediglich leicht erhöht (Opitz, MundKieferGesichtsChir 1998; 2: 122). 
Bedingt durch die Fehlbildungen der Mandibula und die Spaltbildung des Gaumens kommt es zu einer Imbalance zwischen dem Stützgewebe und den knöchernen Strukturen. Dies führt zu einem Kollaps des Weichgewebes mit zurückfallender Zunge, so dass bereits in der Neonatalperiode lebensbedrohliche Atem-, Saug- und Schluckstörungen auftreten, die intensivmedizinische Maßnahmen notwendig machen. Dementsprechend müssen die knöchernen Veränderungen operativ korrigiert werden. Dies erfolgt in der Regel durch eine Distraktionsosteogenese, bei der eine Verlängerung der unterentwickelten Mandibula mit der Folge einer Ventralisierung der Zunge erreicht wird.

Zur präoperativen Planung ist hierfür eine möglichst exakte radiologische Diagnostik notwendig. Von Interesse ist dabei insbesondere der Kiefergelenkwinkel. Weiterhin ist zu klären, ob die Mandibula symmetrisch angelegt ist oder ob eine halbseitige Form des Syndroms vorliegt (Francois et al., Ann ocul 1954; 187: 340). In den hier beschriebenen Fällen wurden ein dosisreduziertes MSCT des Mittelgesichtes und der Mandibula durchgeführt und anschließend 2D- bzw. 3D-Bilder rekonstruiert. Mit einem CT-Datensatz lassen sich dabei alle diagnostischen Fragen im Hinblick auf das Ausmaß kraniofazialer Dysostosen, Gehörgangsatresien und Weichteilbefunde klären. In diesem Zusammenhang bieten Oberflächenrekonstruktionen dem Operateur einen hilfreichen visuellen Eindruck, der durch die interaktive Monitorbetrachtung unterstützt wird. Geometrische Größen wie z.B. der Kiefergelenkwinkel sind exakt bestimmbar (Vannier et al., Radiology 1984; 150: 179). Eine detaillierte Planung der OPStrategie wird durch eine präoperative CT-Untersuchung mit der aktuell verfügbaren Technik deutlich erleichtert. Dabei gilt die Distraktionsosteogenese als eine weithin akzeptierte Methode zur Rekonstruktion einer hypoplastischen Mandibula. Neben der Korrektur der Gesichtsasymmetrie wird hierdurch vor allem die Atemwegsobstruktion beseitigt. Fast immer ist jedoch bei Kindern mit NagerSyndrom mit einer geistigen und körperlichen Entwicklungsverzögerung zu rechnen, die vor allem auf Wachstumsstörungen, Sprachprobleme und Hörverluste zurückzuführen ist.

S. Dammert, M. Funke, H.-A. Merten, Göttingen 